\title{
FORMATION OF DISSIPATIVE STRUCTURES IN LIQUID WATER
}

\author{
E. DEL GIUDICE ${ }^{1} \&$ R.M. PULSELLI ${ }^{2}$ \\ ${ }^{1}$ INFN, Milan, Italy, and International Institute of Biophysics, Neuss, Germany. \\ ${ }^{2}$ Department of Chemistry, University of Siena, Italy.
}

\begin{abstract}
In living systems, water takes part in the dynamics of life, not only because it accounts for $99 \%$ of all biomolecules but also because it provides energy to living matter. Water has the ability to achieve an extended form of organization and provide an ensemble of different coherence domains (CDs) that are phase locked, thus maximizing their capacity to 'look for' energy from the environment. This 'coherence of coherences' of 'biological water' in living systems corresponds to a sort of higher organization. An efficient mechanism of energy transformation from CDs to biomolecules in living matter guarantees the transfer of biochemical energy necessary for the maintenance of life cycles. The dynamics of formation of dissipative structures in liquid water and the process of self-organization of living organisms induced by water are briefly discussed. Dissipative structures appear as a consequence of the phase locking within an ensemble of CDs. The process of charge and discharge of energy is discussed.

Keywords: coherence, dissipative structures, quantum fluctuations, self-organization, water.
\end{abstract}

\section{THERMODYNAMICS AND SELF-ORGANIZATION}

Dynamics of living matter differ deeply from those of non-living matter. The main difference is that inert non-living matter demands a simultaneous supply of external energy to perform work, whereas on the contrary living matter is able to move spontaneously by extracting the necessary energy from its own stock of energy stored over time by metabolism; the organism picks up energy from this reservoir when it is needed. What is the dynamic origin of this difference? Let us recall thermodynamics. The variation of energy $U_{2}-U_{1}$ between the initial state 1 and the final state 2 is the sum of the work $\mathrm{W}$ performed on the system and the heat $\mathrm{Q}$ supplied to it:

$$
\mathrm{U}_{2}-\mathrm{U}_{1}=\mathrm{W}+\mathrm{Q} \text {. }
$$

Since temperature $\mathrm{T}$ is almost constant in biological processes we can express $\mathrm{Q}$ as a function of the variation of entropy.

$$
\mathrm{Q}=\mathrm{T}\left(\mathrm{S}_{2}-\mathrm{S}_{1}\right)
$$

Combining eqns (1) and (2) we get:

$$
\mathrm{W}=\mathrm{U}_{2}-\mathrm{U}_{1}-\mathrm{T}\left(\mathrm{S}_{2}-\mathrm{S}_{1}\right),
$$

which summarizes the requirements of thermodynamics.

In living organisms the dynamics is driven by a decrease of entropy, so that there should be a first stage where the decrease of the thermal energy TS should be transformed into an energy U able to produce work:

$$
\mathrm{T} \Delta \mathrm{S}=\Delta \mathrm{U}<0
$$

This transformation should occur within a 'specialized' structure of the system and produce an energy $\Delta \mathrm{U}$ that, being negative, gets out of the structure. It is possible that this structure could be just water; the process of transformation of energy described earlier is the nucleus of the process

(C) 2010 WIT Press, www.witpress.com

ISSN: 1755-7437 (paper format), ISSN: 1755-7445 (online), http://journals.witpress.com

DOI: 10.2495/DNE-V5-N1-21-26 
of self-organization. The energy $|\Delta U|$ released by the self-organizing structure is received by the 'active' structures of the organism and transformed into work on the environment. Therefore the system should be open.

We now stress a very important point. The energy released from the self-organizing structure is not blind, but is informed energy. Actually this energy arises from a process of concentration from a huge number of degrees of freedom (high entropy) to a smaller set of degrees of freedom (low entropy), but the choice of the degrees of freedom on which the concentration occurs is done by the system itself and is driven by the decrease of entropy. Therefore the direction of the process is dictated by entropy whereas energy, whose flow is driven by the variation of entropy, plays the executive role. In conclusion the possibility of an 'active' matter, and therefore of life, demands the capability of decreasing entropy.

This requirement has been recognized long ago by pioneers such as Schroedinger [1], SzentGyorgyi [2] and Prigogine [3]. Since at that time there was not yet an example of a process internal to the matter able to decrease entropy permanently, Schroedinger introduced the concept of negentropy as a variable whose flow was able to decrease the entropy of the system. However modern Quantum ElectroDynamics (QED) $[4,5]$ has shown that mutual electromagnetic (e.m.) interactions among molecules are able to produce this result from inside the system. We summarize shortly the QED argument.

\section{ELECTRODYNAMICS AND SELF-ORGANISATION}

Atoms and molecules are made up of electrically charged particles (nuclei and electrons) that couple with the e.m. field. These microscopic units may have several configurations, each corresponding to a well-defined value of energy. They settle spontaneously in the lowest energy state (ground state) whereas they can assume the other configurations provided that the necessary difference of energy is supplied from outside, the only possible supplier being the e.m. field. The typical scale of an atom is $1 \AA$ (Angstrom, which corresponds to $10^{-8} \mathrm{~cm}$ ). The typical scale of an energy jump between two different atomic or molecular configurations is about some electronvolts, let say for the sake of definiteness $10 \mathrm{eV}$. This energy should be supplied by a quantum (photon) of the e.m. field, either coming from the ambient e.m. background or emerging from the quantum vacuum. The typical space scale of a photon is its wavelength, which in the case of $10 \mathrm{eV}$ is $1200 \AA$. This means that the volume $\mathrm{V}$ of a photon is a cube whose side is the wavelength.

$$
\mathrm{V}=\lambda^{3}
$$

We realize the existence of a mismatch between the space scales of the matter field (the ensemble of atoms/molecules) and that of the e.m. field. An atom needs an object about a thousand times larger than itself to change its own configuration. Let us start from an ensemble of molecules having a density

$$
\mathrm{n}=\mathrm{N} / \mathrm{V}
$$

where $\mathrm{V}$ is assumed to be the one given by eqn (4).

Let us assume for the sake of simplicity that the atoms have only one excited state with an excitation energy E.

A photon having a wavelength

$$
\lambda=\mathrm{hc} / \mathrm{E},
$$


where $\mathrm{h}$ is the Planck constant and $\mathrm{c}$ the speed of light, coming from the ambient background or the quantum vacuum, excites an atom that, after an interval corresponding to the life time of the excited state, decays releasing back the photon that has two possible fates:

1. to return back where he came from;

2. to excite another atom.

Should the density $\mathrm{n}$ be low, the second option could occur a small number of times only and the photon would eventually join the e.m. field again. However, when the density $\mathrm{n}$ exceeds the threshold $\mathrm{n}_{\text {critical }}$ such that

$$
\mathrm{PVn}_{\text {critical }}=\mathrm{P} \lambda^{3} \mathrm{n}_{\text {critical }}=\mathrm{Pc}^{3} \mathrm{~h}^{3} \mathrm{n}_{\text {critical }} / \mathrm{E}^{3}=1,
$$

where $\mathrm{P}$ is the elementary probability of excitation of an atom by a photon, then the photon cannot revert any longer to its previous state of a quantum of a free e.m. field but remains permanently trapped in the ensemble of atoms.

The same fate occurs to the other photons emerging from the ambient background or the vacuum so that after a short time a large e.m. field is built up within the volume occupied by the ensemble of molecules. This field attracts inside the volume other similar atoms from the surroundings producing an increase of density until the limit density is reached, which corresponds to an interatomic distance equal to the diameter of each atom in the largest configuration involved in the oscillation. The oscillation of atoms between the two configurations and the e.m. oscillation are phase locked and hence this region is called coherence domain (CD). The motion of molecules, which was previously chaotic, now becomes coherent, implying a large decrease of entropy.

The aforementioned dynamics describes exactly the phase transition from a vapour to a liquid and gives a simple example of how a novelty can emerge in a dynamics occurring in an elementary system. Notice also that this phase transition corresponds to a flow of energy from the vacuum to the matter and this breaks the time translation invariance, introducing an internal time of the coherent system. The amount of this flow of energy is controlled by the density of matter and by the strength of coupling of the units of matter to the e.m. field (this parameter is $P$ in eqn (7)).

This scheme holds for all molecular species. However the case of water [6] is peculiar since in this case the coherent oscillation involves an excited configuration at $12.06 \mathrm{eV}$ where there is one electron just below the ionization threshold of $12.60 \mathrm{eV}$. Therefore a CD of water whose size according to eqn (6) is $1000 \AA ̊$ includes a reservoir of quasi-free electrons. Since, according to [6], the statistical weight of the excited configuration in the coherent state is 0.13 and the number of component molecules of the $\mathrm{CD}$ at room temperature is about 5.5 millions, we have about 700,000 quasi-free electrons in each CD. As a consequence, the water CD, different than the CDs of other species, can be further excited. Each supply of external energy, lower than the 'energy gap' that would vaporize the domain, could be received by this ensemble of coherent quasi-free electrons producing a collective motion that because of the coherence would be frictionless [7], so that the lifetime of the induced excitation would be quite long (up to weeks or months; see [8]). The moment of inertia I of the ensemble of the cloud of quasi-free electrons in the $\mathrm{CD}$ is very small, because of the very small mass of the electron. Consequently the excitation energy $\mathrm{L}^{2} / 2 \mathrm{I}$ of the collective excitations ( $\mathrm{L}$ is the angular momentum) could be quite large increasing indefinitely with the angular momentum. Moreover these excited states have a magnetic moment proportional to $\mathrm{L}$ that is aligned by the ambient magnetic field; the earth's magnetic field plays thus a fundamental role.

The long lifetime of these $\mathrm{CD}$ excited states and the alignment of their magnetic moments allows to sum up many excitation-producing states with very large angular momenta and very 
large energies. Water CDs become devices able to pick up high entropy energy from the environment and transform it into low entropy energy stored in the $\mathrm{CD}$. This is the precondition for the formation of a dissipative structure.

\section{THE BIRTH OF A DISSIPATIVE STRUCTURE}

The long lifetime of the excited states of water CDs depends on the inability of CDs to release the energy in a thermal way, since their effective temperature is quite low because of coherence. In order to make possible a CD oscillation, we need to open a channel for the release of energy. This channel is offered actually by the possibility of chemical reactions. Since water molecules cannot react chemically among them, the presence of other molecules is mandatory. As a matter of fact, there is no such thing as pure water; there are always atmospheric gases dissolved into it or even impurities such as ions and other molecules. The particular role of bicarbonates is discussed in ref. [9]. As mentioned in this ref. [9], a bicarbonate aqueous solution is a system able to concentrate environmental chaotic energy and transform it into high grade free energy of photon emission, due to the decrease of its own entropy.

An interesting case is offered by those molecules having in their spectrum an oscillation frequency matching the frequency of oscillation of the water $\mathrm{CD}$, which in its ground state is $0.26 \mathrm{eV}$ corresponding to $6.25 * 10^{13} \mathrm{~Hz}$. These co-resonating molecules can join the collective coherent oscillation of the water molecules in the $\mathrm{CD}$. However the different value of their radiative dipoles (connected with the intensity of the field wavelets each one of them is able to re-emit after being excited) could disrupt the coherence of the ensemble, unless their number would be quite small, say less than $1 \%$ of the water molecules. In fact, in living matter, water molecules account for $99 \%$ of the grand total of the molecules and the earlier argument could provide a rationale for the water dominance in living organisms; a water CD would not be able to govern coherently an ensemble of foreign molecules exceeding the $1 \%$. Under these conditions, the non-aqueous molecules become guest molecules of the $\mathrm{CD}$ and co-owners of the energy stored in them. When this energy increases until reaching the activation energy of the guest molecules, which, different than water molecules, are able to react chemically, the excitation energy is transferred resonantly from the $\mathrm{CD}$ to the guest molecules that, as in a multi-mode laser, discharge the CD producing an ordered pattern of chemical reactions. Since these reactions occur according to a mutual recognition code based on resonance, they develop not randomly but according to a code. This means that the molecular encounters are selective and very fast since they are induced by a field attraction and do not follow a diffusive regime.

According to this interplay between chemistry and electromagnetism, water CDs can enter into an oscillatory regime between their fundamental configuration and one among many excited configurations. Each one of these oscillations can give rise to the onset of a coherence among the CDs [10]. The size of this 'super-domain' is given by the wavelength of the associated e.m. mode, namely by the duration of its oscillation times the speed of light $c$. The duration of oscillation is the consequence of the rate of the energy charge, of the level of the activation energy of the guest molecules and of the rate of the chemical reactions.

However the chemical reactions produce an output of energy that cannot but be assumed by the same coherent region, producing a shift in its frequency of oscillation, which in turn changes the species of guest molecules able to join the coherent oscillation. In this way we gain the possibility of a time dependent biochemistry, where the outcome of a particular cycle opens the way to another different cycle. In each step of this dynamics the environment enters in a fundamental way through the rate of the flow of energy (non-informational parameter) and, more fundamentally, the resonance conditions between molecules and CDs (informational parameter). 
We know, see section 1, that in order to produce coherence we need to release energy outwards, producing disorder beyond the boundaries of the coherent system. Should the system be much too large, the outflow of energy would disrupt its peripheral parts, so that the system should not be larger than its maximal coherence super-domain. It is clear that a decrease of the size of this super-domain would imply a pathology for the whole system.

The analysis of the supercoherent structure of liquid water, and consequently of its self-organization dynamics, is still in an early stage. However the first rough considerations outlined here suggest intriguing convergencies with the results obtained in different frameworks by pioneers such as Prigogine [3], Szent-Gyorgyi [2] and Piccardi [11]. The structure of superdomains shows similarities with the COOS introduced by Tiezzi and Marchettini in this same issue [12]; in particular we stress the appearance in both approaches of an history, of the possibility of an evolution [13]. The concept of supercoherence could also provide a rationale for the peculiar properties exhibited by water undergoing a living dynamics [14] or by interfacial water [15].

At this point we stop writing, imitating Sherazad who became silent when approaching the most telling part of the story.

\section{ACKNOWLEDGEMENTS}

We are grateful to our friends Vittorio Elia, Nadia Marchettini, Elena Napoli, Jerry Pollack, Paola Spinetti, Alberto Tedeschi, Enzo Tiezzi, Giuseppe Vitiello, Vladimir Voeikov, for the passionate discussions and the enthusiasm that made possible the development of this point of view last time and in particular in the Ischia Brainstorming from 24 to 26 May 2009.

\section{REFERENCES}

[1] Schroedinger, E., What is Life? Cambridge University Press: Cambridge, 1944.

[2] Szent-Gyorgyi, A., Introduction to a Supramolecular Biology, Academic Press: New York \& London , 1960.

[3] Nicolis, G. \& Prigogine, I., Self-organization in Non-equilibrium Systems, Wiley \& Sons: New York, 1977.

[4] Preparata, G., QED Coherence in Matter, World Scientific: London, New Jersey, Singapore, 1995.

[5] Del Giudice, E. \& Vitiello, G., Role of the electromagnetic field in the formation of domains in the process of symmetry-breaking phase transition. Physical Review A, 74, 022105 (1-9), 2006.

[6] Arani, R., Bono, I., Del Giudice, E. \& Preparata, G., QED coherence and the thermodynamics of water. International Journal of Modern Physics B, 9, pp. 1813, 1995. doi:10.1142/ S0217979295000744

[7] Del Giudice, E. \& Preparata, G., A new QED picture of water: understanding a few fascinating phenomena. Macroscopic Quantum Coherence, eds E. Sassaroli et al., World Scientific: London, pp. 108-129, 1998.

[8] Elia, V. \& Napoli, E., Dissipative structures in extremely diluted solutions of homeopathic medicines. A molecular model based on physico-chemical and gravimetric evidences. International Journal of Design \& Nature and Ecodynamics, 5(1), pp. 1-10, 2010.

[9] Voeikov, V.L., Ming Ha, D., Mukhitova, O.G., Vilenskaya, N.D., Malishenko, S.I. \& Bogachuk, A.S., Activated bicarbonate solutions as models of ontic open system and prototypes of living respiring systems. International Journal of Design \& Nature and Ecodynamics, 5(1), pp. 30-38, 2010.

[10] Del Giudice, E. \& Tedeschi, A., Water and the autocatalysis in living matter. Electromagnetic Biology and Medicine, 28, pp. 46, 2009. doi:10.1080/15368370802708728 
26 E. Del Giudice \& R.M. Pulselli, Int. J. of Design \& Nature and Ecodynamics. Vol. 5, No. 1 (2010)

[11] Piccardi, G., The Chemical Basis of Medical Climatology, Charles C. Thomas Publisher, Ltd: Springfield, IL, 1962.

[12] Tiezzi, E. \& Marchettini, N., Confined ontic open systems. International Journal of Design \& Nature and Ecodynamics, 5(1), pp. 3-9, 2010.

[13] Del Giudice, E., Pulselli, R.M. \& Tiezzi, E., Thermodynamics of irreversible processes and quantum field theory: an interplay for the understanding of ecosystem dynamics. Ecological Modelling, 220, pp. 1874-1879, 2009. doi:10.1016/j.ecolmodel.2009.04.035

[14] Tedeschi, A., Is the living dynamics able to change the properties of water? International Journal of Design \& Nature and Ecodynamics, 5(1), pp. 60-67, 2010.

[15] Pollack, G.H., Water, Energy and life: fresh views from the water's edge. International Journal of Design \& Nature and Ecodynamics, 5(1), pp. 27-29, 2010. 